\title{
Extracellular Vesicles Released From the Skin Commensal Yeast Malassezia sympodialis Activate Human Primary Keratinocytes
}

\section{OPEN ACCESS}

Edited by:

Thomas Dawson,

Skin Research Institute of Singapore (A*STAR), Singapore

Reviewed by:

Georgios Chamilos,

University of Crete, Greece

Lysangela Ronalte Alves,

Carlos Chagas Institute (ICC), Brazil

${ }^{*}$ Correspondence:

Annika Scheynius annika.scheynius@ki.se

TORCID:

Helen Vallhov orcid.org/0000-0002-9637-55 Annika Scheynius orcid.org/0000-0001-5520-990X

Specialty section:

This article was submitted to

Fungal Pathogenesis,

a section of the journal

Frontiers in Cellular and Infection

Microbiology

Received: 30 September 2019 Accepted: 08 January 2020

Published: 24 January 2020

Citation:

Vallhov $H$, Johansson $C$, Veerman $R E$ and Scheynius A (2020) Extracellular

Vesicles Released From the Skin Commensal Yeast Malassezia sympodialis Activate Human Primary Keratinocytes.

Front. Cell. Infect. Microbiol. 10:6 doi: 10.3389/fcimb.2020.00006

\author{
Helen Vallhov ${ }^{1+}$, Catharina Johansson ${ }^{1}$, Rosanne E. Veerman ${ }^{2}$ and Annika Scheynius ${ }^{1,3 *+}$ \\ ${ }^{1}$ Department of Clinical Science and Education, Karolinska Institutet, and Sachs' Children and Youth Hospital, \\ Södersjukhuset, Stockholm, Sweden, ${ }^{2}$ Immunology and Allergy Unit, Department of Medicine Solna, Karolinska Institutet, \\ and Karolinska University Hospital, Stockholm, Sweden, ${ }^{3}$ Science for Life Laboratory, Karolinska Institutet, Stockholm, \\ Sweden
}

Extracellular vesicles (EVs) released from fungi have been shown to participate in inter-organismal communication and in cross-kingdom modulation of host defense. Malassezia species are the dominant commensal fungal members of the human skin microbiota. We have previously found that Malassezia sympodialis releases EVs. These EVs, designated MalaEx, carry M. sympodialis allergens and induce a different inflammatory cytokine response in peripheral blood mononuclear cells (PBMC) from patients with atopic dermatitis compared to healthy controls. In this study, we explored the host-microbe interaction between MalaEx and human keratinocytes with the hypothesis that MalaEx might be able to activate human keratinocytes to express the intercellular adhesion molecule-1 (ICAM-1, CD54). MalaEx were prepared from $M$. sympodialis (ATCC 42132) culture supernatants by a combination of centrifugation, filtration and serial ultracentrifugation. The MalaEx showed a size range of $70-580 \mathrm{~nm}$ with a mean of $154 \mathrm{~nm}$ using nanoparticle tracking analysis. MalaEx were found to induce a significant up-regulation of ICAM-1 expression on primary human keratinocytes isolated from human ex vivo skin $(p=0.026, n=3)$, compared to the unstimulated keratinocytes. ICAM-1 is a counter ligand for the leukocyte integrins lymphocyte function-associated antigen-1 (LFA-1) and macrophage-1 antigen (Mac-1), of which induced expression on epithelial cells leads to the attraction of immune competent cells. Thus, the capacity of MalaEx to activate keratinocytes with an enhanced ICAM-1 expression indicates an important step in the cutaneous defense against $M$. sympodialis. How this modulation of host cells by a fungus is balanced between the commensal, pathogenic, or beneficial states on the skin in the interplay with the host needs to be further elucidated.

Keywords: extracellular vesicles, fungi, ICAM-1, keratinocytes, MalaEx, Malassezia

\section{INTRODUCTION}

Extracellular vesicles (EVs) are released not only from different mammalian cell-types but also from microorganisms, parasites, and plants (Bielska et al., 2019). EVs are heterogenous in size, 20 up to $1,000 \mathrm{~nm}$ in diameter, and their function differs depending on their cell of origin, different routes involved in biogenesis, release and their cargo (Soares et al., 2017). They are mainly classified into 
two major groups based on size; exosomes of endosomal origin with a diameter up to $150 \mathrm{~nm}$, and microvesicles bigger than $150 \mathrm{~nm}$ generated through outward budding of the plasma membrane (Thery et al., 2018). EVs from microorganisms, such as fungi, are usually around $100-1,000 \mathrm{~nm}$, and have been associated with cytotoxicity, the invasion of host cells, and the transfer of virulence factors (Brown et al., 2015). Fungal EVs have been shown to participate in inter-organismal communication and in cross-kingdom modulation of host cells (Bielska and May, 2019) with their capacity to deliver functional (m)RNAs and micro (mi)RNA-like RNAs to recipient cells (Peres da Silva et al., 2015). Notably, EVs from the host have on their part been found to deliver host sRNA into fungal cells and induce crosskingdom RNA interference (RNAi) to silence fungal virulence genes (Cai et al., 2018).

Malassezia species are the dominant fungal members of the human skin microbiota (Findley et al., 2013) and detectable already on the skin in $100 \%$ of newborns (Nagata et al., 2012). The genus Malassezia comprises a heterogenous group of increasing number of identified species, and novel culture independent methods suggest a wider-spread than previously described (Theelen et al., 2018). Although, a commensal skin colonizing yeast, Malassezia are also associated with a number of skin disorders including pityriasis versicolor, folliculitis, seborrheic dermatitis, dandruff, and atopic dermatitis (AD) (Saunders et al., 2012). Recently, it was found that Malassezia restricta is associated with the colonic mucosa in a subset of patients with Chrohn's disease who have a disease-linked polymorphism in CARD9 (Limon et al., 2019). This observation is interesting in a host-microbe perspective indicating that host genetic factors may increase colonization with Malassezia and the inflammatory response.

For many years, we have utilized Malassezia sympodialis, the fungi most frequently colonizing the skin of both $\mathrm{AD}$ patients and healthy individuals in our part of the globe (Sandstrom Falk et al., 2005), as a model to investigate host-microbe interactions. We have discovered that M. sympodialis releases EVs, which we designated MalaEx (Gehrmann et al., 2011). These EVs are compared to their parental $M$. sympodialis whole yeast cells enriched in 110 proteins, among those two of the $M$. sympodialis allergens, Mala s 1 and Mala s 7 (Johansson et al., 2018), and they can induce inflammatory cytokine responses with a significantly higher IL-4 production in peripheral blood mononuclear cells (PBMC) from patients with AD sensitized to Malassezia compared to healthy controls (Gehrmann et al., 2011). Thus, MalaEx seem to play a contributing role in eliciting and maintaining eczema in patients with AD. Furthermore, we have in MalaEx detected several small RNAs with well-defined start and stop positions in a length range of 16-22 nucleotides (Rayner et al., 2017), suggesting their capacity to deliver either autocrine or paracrine signaling for M. sympodialis in the interplay with the host and the environment.

The first major cell population Malassezia interacts with on the skin is epidermal keratinocytes. Besides forming an effective mechanical barrier to the outer environment keratinocytes are also active components of the immunoregulatory network in the skin (Di Meglio et al., 2011). Keratinocytes produce and express several mediators, in response to outer signals and transmit those to immune cells in the skin thereby regulating skin immunity and inflammation (Pasparakis et al., 2014). Whole yeast cells from different Malassezia species have been found to induce release of a variable profile of inflammatory mediators by keratinocytes (Watanabe et al., 2001; Ishibashi et al., 2006; Donnarumma et al., 2014). Previously, we discovered an active binding and internalization of MalaEx into human keratinocytes, where MalaEx were mainly found in close proximity of the keratinocyte nuclei, suggesting a central communication with the host cell (Johansson et al., 2018). In this study, we explored the hostmicrobe interaction between MalaEx and human keratinocytes with the hypothesis that MalaEx might be able to activate human keratinocytes to express the intercellular adhesion molecule-1 (ICAM-1, CD54).

\section{MATERIALS AND METHODS}

Malassezia sympodialis Culture Conditions M. sympodialis (ATCC 42132) was cultured on Dixon agar plates (Gueho et al., 1996) modified to contain 1\% (vol/vol) Tween 60, 1\% (wt/vol) agar, and no oleic acid (mDixon) at $32^{\circ} \mathrm{C}$. After 2 or 4 days, the yeast cells were harvested, resuspended and washed in PBS. Counting in a Bürker chamber using trypan blue exclusion showed a viability above 95\%. From the 4 days cultures $6 \times 10^{7}$ live yeast cells $/ \mathrm{ml}$ were added to RPMI 1640 medium supplemented with penicillin 100 units $/ \mathrm{ml}$, streptomycin $100 \mu \mathrm{g} / \mathrm{ml}, 2 \mathrm{mM}$ L-glutamine, and $10 \%$ heat inactivated fetal calf serum (all from Gibco BRL, Life Technologies Ltd, Paisley, UK) and incubated for $48 \mathrm{~h}$ in $6 \% \mathrm{CO}_{2}$ at $37^{\circ} \mathrm{C}$, as previously described (Gehrmann et al., 2011). Prior usage, fetal calf serum had been ultra centrifuged overnight at $100000 \times \mathrm{g}$ followed by filtration through a $0.22 \mu \mathrm{m}$ filter (Nordic Biolabs, Täby, Sweden) to remove possible EV contaminants. Each culture batch consisted of $320 \mathrm{ml}$ distributed over four $175 \mathrm{~cm}^{2}$ flasks (Falcon, Corning Inc., Tewksbury, MA, USA). At each culture step blood and Sabouraud agar plates were inoculated in parallel to exclude bacterial and Candida contaminations, respectively.

\section{MalaEx Preparation}

MalaEx were prepared from the $48 \mathrm{~h}$ M. sympodialis culture supernatants by using a combination of centrifugation, filtration, and serial ultracentrifugation. The culture supernatants were spun at $1200 \times \mathrm{g}$ for $5 \mathrm{~min}$ followed by $3000 \times \mathrm{g}$ for $30 \mathrm{~min}$, and thereafter filtered through a $0.22 \mu \mathrm{m}$ filter (Nordic Biolabs) and frozen to $-80^{\circ} \mathrm{C}$ until further preparation. After thawing at RT, supernatants were centrifuged at $10000 \times \mathrm{g}$ for $30 \mathrm{~min}$. Thereafter, MalaEx were pelleted from the supernatants at 100 $000 \times \mathrm{g}$ for $90 \mathrm{~min}$, re-suspended in PBS and pelleted again at $100000 \times \mathrm{g}$ for $90 \mathrm{~min}$. The resulting pellet was carefully resuspended in $100 \mu \mathrm{l} \mathrm{PBS}$ and stored frozen at $-80^{\circ} \mathrm{C}$. To avoid batch variations and to obtain enough MalaEx for the stimulation experiments (see below) three MalaEx preparations were pooled. The protein content was measured using a detergent compatible (DC) protein assay according to the manufacturer's instructions (BioRad, Hercules, CA, USA). 


\section{Nanoparticle-Tracking Analysis (NTA)}

The particle size and concentration of the pooled MalaEx preparation was measured using a LM10 platform with sCMOS camera from NanoSight Ltd, Amesbury, UK. The system is equipped with a $405 \mathrm{~nm}$ laser running nanoparticle tracking analysis (NTA) 2.3 analytical software package. The samples were diluted $3,000 \times$ in $30 \mathrm{kDa}$ filtered PBS and analyzed with camera level 14 and detection threshold 7. Four consecutive videos were recorded in $\mathrm{RT}$ while injecting the sample with a syringe pump (speed 50). The result is expressed as the mean particle size \pm SD and the particle concentration of the four separate NTA runs of the pooled MalaEx sample.

\section{Preparation of Human Primary Keratinocytes}

Epidermal keratinocytes were isolated according to the manufacturer's instructions (Gibco Invitrogen Corporation, Paisley, UK) from human abdomen ex vivo skin received from a local plastic surgery clinic. In short, thin skin tissues were prepared by using a dermatome, which thereafter were incubated in a Dispase solution (25 caseinolytic units/ml; Gibco Invitrogen Corporation) for $18 \mathrm{~h}$ at $4^{\circ} \mathrm{C}$. Epidermis was separated from dermis and placed into $0.05 \%$ Trypsin-EDTA (Gibco Invitrogen Corporation) for $15 \mathrm{~min}$ at $37^{\circ} \mathrm{C}$ for cell dissociation. After addition of Soybean Trypsin Inhibitor (Gibco Invitrogen Corporation) at a concentration of $10 \mathrm{mg} / \mathrm{ml}$, cells were pelleted, washed and suspended in complete serum-free keratinocyte medium supplemented with $100 \mathrm{IU} / \mathrm{ml}$ penicillin, $100 \mu \mathrm{g} / \mathrm{ml}$ streptomycin, and $0.5 \mu \mathrm{g} / \mathrm{ml}$ Amphotericin B (Gibco Invitrogen Corporation). Approximately $3 \times 10^{6}$ cells, with a viability above 95\% using trypan blue exclusion, were seeded in $15 \mathrm{ml}$ culture medium in each T-75 culture flask (Falcon ${ }^{\circledR}$, Corning Life Sciences, Tweksburry, MA, USA) and cultured at $37^{\circ} \mathrm{C}, 6 \%$ $\mathrm{CO}_{2}$. The culture medium was replaced every second or third day until the cell confluence reached $\sim 75 \%$, within $19-28$ days depending on skin donor. The cells were then detached with $0.05 \%$ Trypsin-EDTA treatment for $5 \mathrm{~min}$ at $37^{\circ} \mathrm{C}$, followed by Soybean Trypsin Inhibitor $10 \mathrm{mg} / \mathrm{ml}$, washed in culture medium, and thereafter $\sim 5 \times 10^{6}$ cells in $2 \mathrm{ml}$ per vial were frozen using $10 \%$ DMSO and $50 \%$ heat inactivated fetal calf serum depleted from EV by ultracentrifugation and stored at $-150^{\circ} \mathrm{C}$.

\section{Co-cultures of $\boldsymbol{M}$. sympodialis or MalaEx With Human Primary Keratinocytes}

After thawing in a $37^{\circ} \mathrm{C}$ water bath for $1 \mathrm{~min}$, the keratinocytes were transferred to a $50 \mathrm{ml}$ tube and $18 \mathrm{ml}$ of culture medium was slowly added. Thereafter, the keratinocytes were spun down, $180 \times \mathrm{g}$ for $7 \mathrm{~min}$, and re-suspended in culture medium. Approximately $3 \times 10^{6}$ cells, viability $80-90 \%$, were seeded in $15 \mathrm{ml}$ culture medium in each T-75 culture flask and cultured as above until the cell confluence, after 7-14 days, had reached $\sim 75 \%$. The cells were detached with $0.05 \%$ Trypsin-EDTA (see above), counted and seeded into $\mu$-slides with 8 wells, $1 \mathrm{~cm}^{2}$ growth area per well, with a glass coverslip bottom (Cat. No. 80827, Ibidi, Martinsried, Germany). $0.6 \times 10^{5}$ keratinocytes in $0.35 \mathrm{ml}$ culture medium were seeded into each well. The $\mu$-slides were incubated for $2-4$ days at $37^{\circ} \mathrm{C}, 6 \% \mathrm{CO}_{2}$ until $75 \%$ confluency was reached. Thereafter, the culture medium was removed and replaced with $0.35 \mathrm{ml}$ fresh medium, and the stimulation agents were added to the keratinocytes; $M$. sympodialis $\left(0.6 \times 10^{5}\right.$ and $3 \times 10^{5}$ live yeast cells/well $)$ harvested from the mDixon agar plates after 2 days of culture and the pooled MalaEx $(1,10$, and $50 \mu \mathrm{g} / \mathrm{ml})$. Lipopolysaccharides (LPS) (10 $\mu \mathrm{g} / \mathrm{ml}, \mathrm{L} 8274$, Sigma-Aldrich, Steinheim, Germany) was used as a positive control (Marcatili et al., 1997) and keratinocytes cultured in only the medium as a negative control. The cultures were incubated at $37^{\circ} \mathrm{C}, 6 \% \mathrm{CO}_{2}$ for $24 \mathrm{~h}$. The cells in the $\mu$-slides were used for further analyses (see below).

\section{Confocal Laser-Scanning Microscopy (CLSM)}

The keratinocytes co-cultured with M. sympodialis, MalaEx or LPS, or cultured alone in the 8 well $\mu$-slides with a glass coverslip bottom were directly fixed in $4 \%$ formaldehyde for $10 \mathrm{~min}$, treated with $0.5 \%$ Triton-X-100 (BDH Laboratory Supplies, Poole, UK) for $5 \mathrm{~min}$, and blocked with $5 \%$ bovine serum albumin (BSA, Sigma-Aldrich) for $5 \mathrm{~min}$ at RT. Thereafter, Alexa Fluor 488 mouse anti-CD54/ICAM-1 or Alexa Fluor $488 \mathrm{IgG}_{1}$ isotype control (Biolegend, San Diego, CA, USA), both at 1:20 dilution in 5\% BSA, were added for $1 \mathrm{~h}$ at RT. The glass coverslip bottoms were finally covered with Prolong Gold antifade mountant (Invitrogen, Thermo Fisher Scientific, MA, USA). Fluorescent images as z-scans and phase contrast images were acquired on a CLSM (TCS SP2; Leica Microsystems, Mannheim, Germany). Each florescent image was combined with each corresponding phase contrast image. Confocal images were used to manually calculate the $\%$ of ICAM- 1 positive keratinocytes defined as strongly positive. For each culture condition 100 cells were analyzed.

\section{Statistical Analysis}

Statistical differences of stimulated keratinocytes compared to unstimulated was assessed by paired $t$-test using GraphPad Prism software (GraphPad Software, Inc. https://www.graphpad. com/scientific-software/prism/). Data are expressed as mean \pm standard error of the mean (SEM). Differences were considered significant when $p<0.05$.

\section{RESULTS}

\section{Characterization of MalaEx}

The pooled batch of three MalaEx batches harvested from $48 \mathrm{~h}$ M. sympodialis culture supernatants had a protein content of $1.45 \mathrm{mg} / \mathrm{ml}$. NTA analysis indicated that the MalaEx had a size range of $70-580 \mathrm{~nm}$, with a mean of $154 \mathrm{~nm}(n=4$ video recordings) and the total particle concentration was $1.19 \times 10^{13}$ particles/ml (Figure 1).

\section{MalaEx Induce ICAM-1 Expression of Keratinocytes}

Co-cultures of keratinocytes with MalaEx was performed for $24 \mathrm{~h}$ on $\mu$-slides with 8 wells and a glass coverslip bottom enabling direct analysis with CLSM. Unstimulated keratinocytes showed 


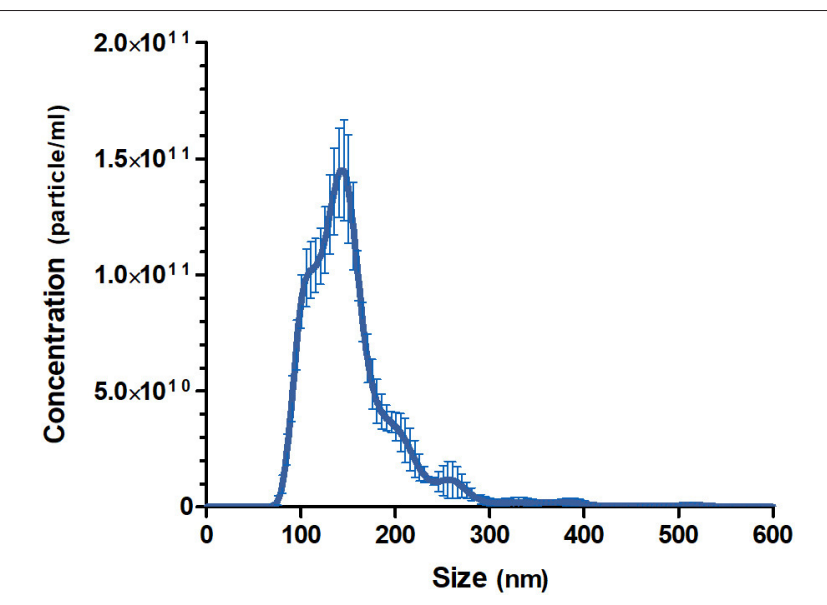

FIGURE 1 | Size distributions and particle concentration of the pooled MalaEx preparation. Three MalaEx preparations were pooled and the pooled sample was investigated by nanoparticle tracking analysis (NTA) using NanoSight. The mean particle size and the particle concentration of four separate NTA runs of the pooled MalaEx sample are plotted. Error bars represent SD.

that around $2 \%$ of the cells had a strong expression of ICAM1 (Figure 2). MalaEx were found to induce an intense ICAM-1 expression on around $5-22 \%$ of the keratinocytes from the three different skin donors with a significant difference for the higher concentration of MalaEx, $10 \mu \mathrm{g} / \mathrm{ml}$ (mean $\pm \mathrm{SEM}, 13.7 \pm 1.8 \%$, $p=0.026$ ), as did the positive LPS control (mean $26 \pm 3.6 \%, p=$ 0.024 ), compared to the unstimulated keratinocytes (Figure 2). We also tested addition of $50 \mu \mathrm{g} / \mathrm{ml}$ of MalaEx without any additional increase in ICAM-1 expression on the keratinocytes (data not shown). Co-culture with $M$. sympodialis whole yeast cells $\left(0.6 \times 10^{5}\right.$ cells/well $)$ resulted in an upregulation of ICAM-1 expression to $8-15 \%$ of the keratinocytes from two of the donors; the donor who did not respond was the same who had a low response to $1 \mu \mathrm{g} / \mathrm{ml}$ of MalaEx (Figure 2). The 5 -fold higher concentration, $3 \times 10^{5}$ live yeast cells/well, gave similar results (data not shown).

\section{DISCUSSION}

The microbial flora on the skin constantly interact with the host skin barrier including immune competent cells in a complex manner influencing local and systemic immunity (Pasparakis et al., 2014). In the current study, we addressed whether nanovesicles, MalaEx, released by a commensal yeast on the skin, M. sympodialis, have capacity to modify keratinocytes. We found that MalaEx can activate keratinocytes to increase the expression of ICAM-1.

The MalaEx preparation harvested by a combination of centrifugation, filtration, and serial ultracentrifugation from supernatants from M. sympodialis had a size range of 70$580 \mathrm{~nm}$ in diameter, with a mean of $154 \mathrm{~nm}$, similar to our previous reports on MalaEx (Rayner et al., 2017; Johansson et al., 2018). Our data are comparable with other studies on fungal EVs considering that different protocols for isolation of
EVs and the various methods used for their analysis contribute to variations in reported sizes of fungal EVs (see Table 1 in Bielska and May, 2019). With cryo-electron tomography we have previously demonstrated that MalaEx have different sizes and are morphological diverse with varying electron-dense material suggesting different amount of internal content (Johansson et al., 2018). The heterogeneity of EVs most likely reflects distinct mechanisms of biogenesis, release, and functions (Soares et al., 2017). Our previous observation that MalaEx were mainly found in close proximity of the keratinocyte nuclei after internalization in keratinocytes, using super-resolution fluorescence 3D-SIM imaging, suggested a microbe-host communication (Johansson et al., 2018). We therefore in the present study investigated the expression of the activation marker ICAM-1 on the keratinocytes after co-cultures with MalaEx.

ICAM-1 is the best characterized inducible adhesion molecule on epithelial cells and is a counter ligand for the leukocyte integrins lymphocyte function-associated antigen-1 (LFA-1) and macrophage-1 antigen (Mac-1) (Smith et al., 1989). In the skin enhanced ICAM-1 expression on keratinocytes precedes dermal T lymphocytic infiltration (Griffiths and Nickoloff, 1989), and treatment with monoclonal antibodies to ICAM-1 or LFA1 inhibits cell infiltration in contact sensitivity reactions in sensitized mice (Scheynius et al., 1993). We here found that only around $2 \%$ of the keratinocytes had a strong expression of ICAM1 in the unstimulated cultures, in agreement with low constitutive expression of ICAM-1 on human keratinocytes (Dustin et al., 1988). MalaEx were capable to induce a 6-fold increase of strong ICAM-1 expression on primary human keratinocytes, and two of the keratinocyte donors responded also to M. sympodialis whole yeast cells with increased ICAM-1 expression compared to the unstimulated keratinocytes (Figure 2). Inducible ICAM1 expression on human keratinocytes is highly variable between different donors (Middleton and Norris, 1995), which can explain why one donor, $\mathrm{nr} 2$, did not respond to the concentration $M$. sympodialis whole yeast cells used, nor to the lower concentration of MalaEx (Figure 2). Further studies will explore the donor variation in response to MalaEx.

Increased ICAM-1 expression on human keratinocytes can be induced by LPS (Marcatili et al., 1997), IFN-r(Dustin et al., 1988), TNF- $\alpha$, and ultraviolet radiation (Krutmann et al., 1990). We could detect significantly increased ICAM-1 expression on the keratinocytes when the keratinocytes were co-cultured with MalaEx or LPS compared to the unstimulated keratinocytes (Figure 2). The kinetics and molecular mechanisms underlying the MalaEx induced upregulation of ICAM-1 expression on the keratinocytes are presently unclear and also whether other EVs (Bielska et al., 2019), have similar capacity. A recent review article highlights that our knowledge of the comparability of EVs in connecting different kingdoms is limited (Woith et al., 2019). Regarding capacity to participate in an allergic immune response we have previously, however, demonstrated that $M$. sympodialis released MalaEx can carry allergens similar to human dendritic or B-cell-derived exosomes and that all three types of EVs could induce Th2like cytokine responses in blood cells from allergic donors (Admyre et al., 2007; Gehrmann et al., 2011; Vallhov et al., 2015). 


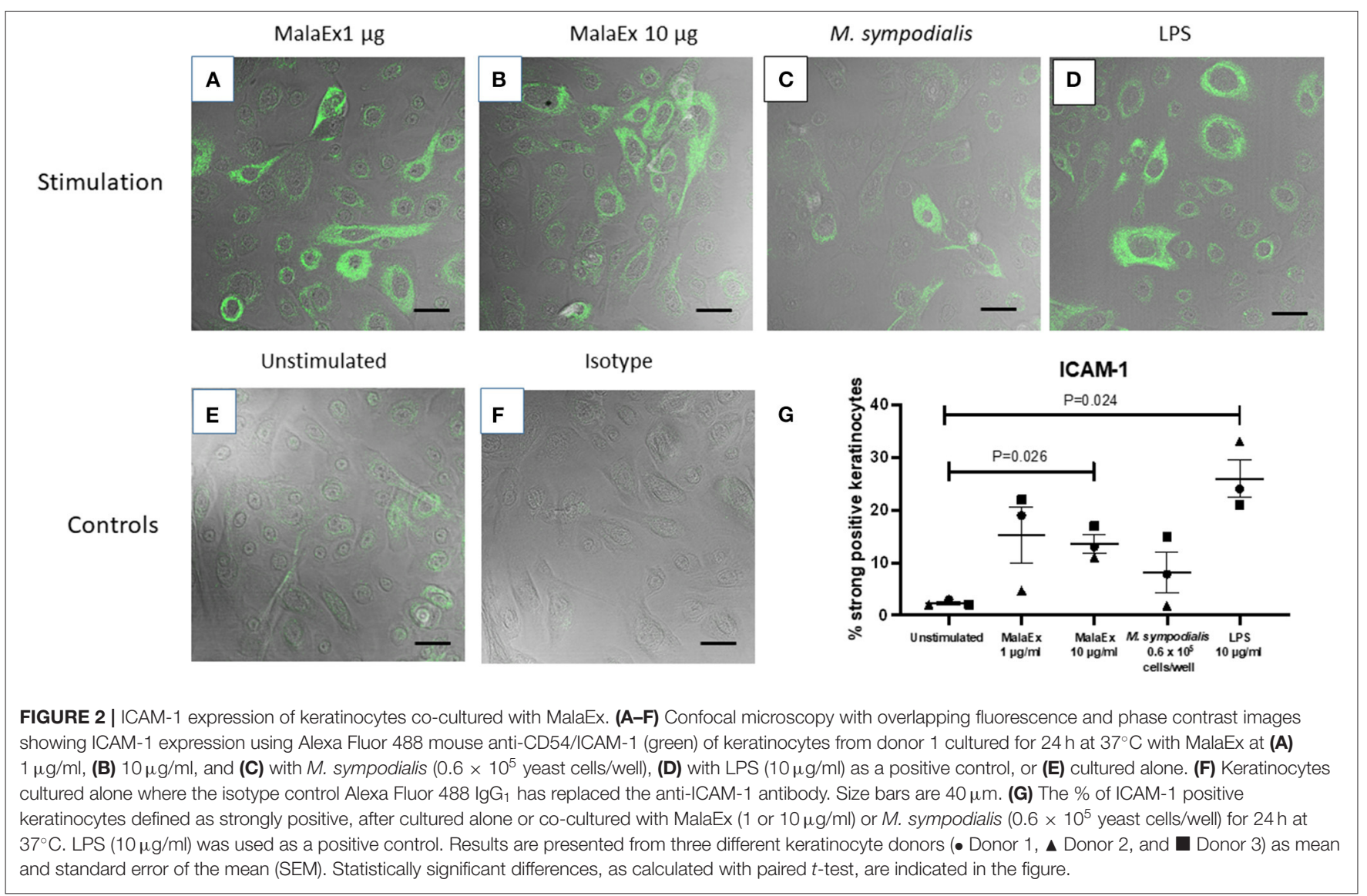

A strength in the present study is the decision to mimic the situation on human skin as much as possible. We therefore used human primary keratinocytes from different donors, all in early passage. Cell lines, can behave considerable different compared with primary keratinocytes (Middleton and Norris, 1995), which is also the case for murine keratinocytes (Grone, 2002), and animal models with anatomical and immunological differences compared with humans (Di Meglio et al., 2011). At the same time our decision was a challenge, since the number of skin donors was limited, they were to us anonymous, and variations between human donors is expected (Middleton and Norris, 1995). For a more in vivo like situation, we also included the mixture of different MalaEx released into the supernatants of M. sympodialis, all assumed to be present on the skin. To obtain enough MalaEx we made a pool to avoid bias between different batches. Another strength is that the co-cultures of keratinocytes with MalaEx was performed on $\mu$-slides with a glass coverslip bottom allowing direct analysis with CLSM, whereby in vitro manipulations to detach and separate the keratinocytes for further analysis were avoided. A limitation is, however, the use of mono-layer cultures of keratinocytes not reflecting the structure with distinct different layers of keratinocytes and appendages in normal skin. In future studies, human skin explants could be considered as a suitable model for superficial fungal infections (Corzo-Leon et al., 2019).
The ratio of Malassezia cells or their EVs to the number of keratinocytes in different in vitro studies are difficult to compare and relate to the complex in vivo situation in healthy vs. inflammatory skin conditions. To unravel the presence and biological significance of different EVs in vivo detailed studies are needed requiring development of new technologies (Coelho and Casadevall, 2019; Margolis and Sadovsky, 2019). In agreement with our observation that keratinocytes can be activated by fungal EVs in vitro is a study where EVs from the dermatophyte Trichophyton interdigitale induced the release of proinflammatory mediators by the human keratinocyte line HaCat after 24 co-culture (Bitencourt et al., 2018). Recently, EVs from Malassezia furfur were found capable to stimulate IL6 production in $\mathrm{HaCaT}$ cells and mice epidermal keratinocytes (Zhang et al., 2019), strongly supporting our results that Malassezia EVs, not only their parental whole yeast cells (Watanabe et al., 2001; Ishibashi et al., 2006; Donnarumma et al., 2014), have the capacity to activate keratinocytes. Furthermore, they presented promising in vivo evidence in a mouse model showing that EVs from $M$. furfur topically applied could penetrate the skin and induce IL-6 expression on the keratinocytes as analyzed with immunohistochemical staining on skin sections (Zhang et al., 2019). Notably, they also confirmed our observation (Johansson et al., 2018) on internalization of EVs by keratinocytes and their perinuclear distribution pattern (Zhang et al., 2019). 
Potential beneficial effects to harbor the commensal yeast Malassezia on the skin should not be neglected to elucidate. In that line, a unique secreted as partyl protease produced by Malassezia globosa, MgSAP1, was discovered to hinder Staphylococcus aureus biofilm formation, an established virulence attribute of S. aureus ( $\mathrm{Li}$ et al., 2018). This study invites for investigations if fungal EVs have a role in release and transport of such molecules in microbial interactions and their possible beneficial effects on the host (Ianiri et al., 2018).

\section{CONCLUSIONS}

In conclusion, our data show that $M$. sympodialis released nanosized MalaEx are able to activate human keratinocytes with an enhanced ICAM-1 expression. This implies a possible cross-kingdom modulation of host cells where activation of keratinocytes by MalaEx may be an important first step in cutaneous defense to M. sympodialis. How this modulation of host cells by a fungus is balanced between the commensal, pathogenic or beneficial states on the skin in the interplay with the host needs to be further elucidated.

\section{DATA AVAILABILITY STATEMENT}

The raw data supporting the conclusions of this article will be made available by the authors, without undue reservation, to any qualified researcher.

\section{ETHICS STATEMENT}

The study was approved by the regional ethical review board in Stockholm (2015/2082-31/1). Written informed consent was obtained at Strandkliniken, a local plastic surgery clinic in

\section{REFERENCES}

Admyre, C., Bohle, B., Johansson, S. M., Focke-Tejkl, M., Valenta, R., Scheynius, A., et al. (2007). B cell-derived exosomes can present allergen peptides and activate allergen-specific T cells to proliferate and produce TH2-like cytokines. J. Allergy Clin. Immunol. 120, 1418-1424. doi: 10.1016/j.jaci.2007.06.040

Bielska, E., Birch, P. R. J., Buck, A. H., Abreu-Goodger, C., Innes, R. W., Jin, H., et al. (2019). Highlights of the mini-symposium on extracellular vesicles in inter-organismal communication, held in Munich, Germany, August 2018. J. Extracell Vesicles 8:1590116. doi: 10.1080/20013078.2019.1590116

Bielska, E., and May, R. C. (2019). Extracellular vesicles of human pathogenic fungi. Curr. Opin. Microbiol. 52, 90-99. doi: 10.1016/j.mib.2019.05.007

Bitencourt, T. A., Rezende, C. P., Quaresemin, N. R., Moreno, P., Hatanaka, O., Rossi, A., et al. (2018). Extracellular vesicles from the dermatophyte Trichophyton interdigitale modulate macrophage and keratinocyte functions. Front. Immunol. 9:2343. doi: 10.3389/fimmu.2018.02343

Brown, L., Wolf, J. M., Prados-Rosales, R., and Casadevall, A. (2015). Through the wall: extracellular vesicles in Gram-positive bacteria, mycobacteria and fungi. Nat. Rev. Microbiol. 13, 620-630. doi: 10.1038/nrmicro3480

Cai, Q., Qiao, L., Wang, M., He, B., Lin, F. M., Palmquist, J., et al. (2018). Plants send small RNAs in extracellular vesicles to fungal pathogen to silence virulence genes. Science 360, 1126-1129. doi: 10.1126/science.aar4142

Coelho, C., and Casadevall, A. (2019). Answers to naysayers regarding microbial extracellular vesicles. Biochem. Soc. Trans. 47, 1005-1012. doi: 10.1042/BST20180252
Stockholm, from all subjects donating skin. The donors were anonymous to the recipients of the skin. All experiments were performed in accordance with the Helsinki Declaration ethical principles for medical research.

\section{AUTHOR CONTRIBUTIONS}

$\mathrm{HV}, \mathrm{CJ}$, and AS conceptualized and designed the study. HV and CJ performed the co-culture experiments. RV did the NTA analysis. CJ worked on the statistical analysis. AS wrote the manuscript together with HV. CJ and RV wrote sections of the manuscript. All authors contributed to manuscript revision, read, and approved the submitted version.

\section{FUNDING}

This study was supported by grants from the Swedish Research Council, the Swedish Asthma and Allergy Association's Research Foundation, the Hesselman Foundation, HKH Crown Princess Lovisa's Association for Child Healthcare, and The Swedish Cancer- and Allergy Fund.

\section{ACKNOWLEDGMENTS}

We thank Strandkliniken, Stockholm, and their patients for supplying us with skin, and Daniel Jans, Superresolution Microscopy Support, Advanced Light Microscopy Facility, Royal Institute of Technology, Science for Life Laboratory Solna, Sweden, for advice how to select culture wells for CLSM, and Carol A. Munro, Dora E. Corzo-León, and Donna M. MacCallum, MRC Center for Medical Mycology at the University of Aberdeen, Institute of Medial Sciences, Aberdeen, United Kingdom, for constructive discussions.

Corzo-Leon, D. E., Munro, C. A., and MacCallum, D. M. (2019). An ex vivo human skin model to study superficial fungal infections. Front. Microbiol. 10:1172. doi: 10.3389/fmicb.2019.01172

Di Meglio, P., Perera, G. K., and Nestle, F. O. (2011). The multitasking organ: recent insights into skin immune function. Immunity. 35, 857-869. doi: 10.1016/j.immuni.2011.12.003

Donnarumma, G., Perfetto, B., Paoletti, I., Oliviero, G., Clavaud, C., Del Bufalo, A., et al. (2014). Analysis of the response of human keratinocytes to Malassezia globosa and restricta strains. Arch. Dermatol. Res. 306, 763-768. doi: 10.1007/s00403-014-1479-1

Dustin, M. L., Singer, K. H., Tuck, D. T., and Springer, T. A. (1988). Adhesion of T lymphoblasts to epidermal keratinocytes is regulated by interferon gamma and is mediated by intercellular adhesion molecule 1 (ICAM-1). J. Exp. Med. 167, 1323-1340. doi: 10.1084/jem.167.4.1323

Findley, K., Oh, J., Yang, J., Conlan, S., Deming, C., Meyer, J. A., et al. (2013). Topographic diversity of fungal and bacterial communities in human skin. Nature 498, 367-370. doi: 10.1038/nature12171

Gehrmann, U., Qazi, K. R., Johansson, C., Hultenby, K., Karlsson, M., Lundeberg, L., et al. (2011). Nanovesicles from Malassezia sympodialis and host exosomes induce cytokine responses-novel mechanisms for host-microbe interactions in atopic eczema. PLoS ONE 6:e21480. doi: 10.1371/journal.pone.00 21480

Griffiths, C. E., and Nickoloff, B. J. (1989). Keratinocyte intercellular adhesion molecule-1 (ICAM-1) expression precedes dermal T lymphocytic infiltration in allergic contact dermatitis (Rhus dermatitis). Am. J. Pathol. 135, 1045-1053. 
Grone, A. (2002). Keratinocytes and cytokines. Vet. Immunol. Immunopathol. 88, 1-12. doi: 10.1016/S0165-2427(02)00136-8

Gueho, E., Midgley, G., and Guillot, J. (1996). The genus Malassezia with description of four new species. Antonie Van Leeuwenhoek 69, 337-355. doi: $10.1007 / \mathrm{BF} 00399623$

Ianiri, G., Heitman, J., and Scheynius, A. (2018). The skin commensal yeast Malassezia globosa thwarts bacterial biofilms to benefit the host. J. Invest. Dermatol. 138, 1026-1029. doi: 10.1016/j.jid.2018.01.008

Ishibashi, Y., Sugita, T., and Nishikawa, A. (2006). Cytokine secretion profile of human keratinocytes exposed to Malassezia yeasts. FEMS Immunol. Med. Microbiol. 48, 400-409. doi: 10.1111/j.1574-695X.2006.00163.x

Johansson, H. J., Vallhov, H., Holm, T., Gehrmann, U., Andersson, A., Johansson, C., et al. (2018). Extracellular nanovesicles released from the commensal yeast Malassezia sympodialis are enriched in allergens and interact with cells in human skin. Sci. Rep. 8:9182. doi: 10.1038/s41598-018-27451-9

Krutmann, J., Kock, A., Schauer, E., Parlow, F., Moller, A., Kapp, A., et al. (1990). Tumor necrosis factor beta and ultraviolet radiation are potent regulators of human keratinocyte ICAM-1 expression. J. Invest. Dermatol. 95, 127-131. doi: 10.1111/1523-1747.ep12477839

Li, H., Goh, B. N., Teh, W. K., Jiang, Z., Goh, J. P. Z., Goh, A., et al. (2018). Skin commensal Malassezia globosa secreted protease attenuates Staphylococcus aureus biofilm formation. J. Invest. Dermatol. 138, 1137-1145. doi: 10.1016/j.jid.2017.11.034

Limon, J. J., Tang, J., Li, D., Wolf, A. J., Michelsen, K. S., Funari, V., et al. (2019). Malassezia is associated with Crohn's disease and exacerbates colitis in mouse models. Cell Host Microbe 25, 377-388.e6. doi: 10.1016/j.chom.2019.01.007

Marcatili, A., Cipollaro de l'Ero, G., Galdiero, M., Folgore, A., and Petrillo, G. (1997). TNF-alpha, IL-1 alpha, IL-6 and ICAM-1 expression in human keratinocytes stimulated in vitro with Escherichia coli heat-shock proteins. Microbiology 143 (Pt 1), 45-53. doi: 10.1099/00221287-143-1-45

Margolis, L., and Sadovsky, Y. (2019). The biology of extracellular vesicles: the known unknowns. PLoS Biol. 17:e3000363. doi: 10.1371/journal.pbio.3000363

Middleton, M. H., and Norris, D. A. (1995). Cytokine-induced ICAM-1 expression in human keratinocytes is highly variable in keratinocyte strains from different donors. J. Invest. Dermatol. 104, 489-496. doi: 10.1111/1523-1747.ep12605923

Nagata, R., Nagano, H., Ogishima, D., Nakamura, Y., Hiruma, M., and Sugita, T. (2012). Transmission of the major skin microbiota, Malassezia, from mother to neonate. Pediatr. Int. 54, 350-355. doi: 10.1111/j.1442-200X.2012.03563.x

Pasparakis, M., Haase, I., and Nestle, F. O. (2014). Mechanisms regulating skin immunity and inflammation. Nat. Rev. Immunol. 14, 289-301. doi: $10.1038 /$ nri3646

Peres da Silva, R., Puccia, R., Rodrigues, M. L., Oliveira, D. L., Joffe, L. S., Cesar, G. V., et al. (2015). Extracellular vesicle-mediated export of fungal RNA. Sci. Rep. 5:7763. doi: 10.1038/srep07763

Rayner, S., Bruhn, S., Vallhov, H., Andersson, A., Billmyre, R. B., and Scheynius, A. (2017). Identification of small RNAs in extracellular vesicles from the commensal yeast Malassezia sympodialis. Sci. Rep. 7:39742. doi: $10.1038 /$ srep39742

Sandstrom Falk, M. H., Tengvall Linder, M., Johansson, C., Bartosik, J., Back, O., Sarnhult, T., et al. (2005). The prevalence of Malassezia yeasts in patients with atopic dermatitis, seborrhoeic dermatitis and healthy controls. Acta Derm. Venereol. 85, 17-23. doi: 10.1080/00015550410022276
Saunders, C. W., Scheynius, A., and Heitman, J. (2012). Malassezia fungi are specialized to live on skin and associated with dandruff, eczema, and other skin diseases. PLoS Pathog. 8:e1002701. doi: 10.1371/journal.ppat.1002701

Scheynius, A., Camp, R. L., and Pure, E. (1993). Reduced contact sensitivity reactions in mice treated with monoclonal antibodies to leukocyte functionassociated molecule-1 and intercellular adhesion molecule-1. J. Immunol. $150,655-663$.

Smith, C. W., Marlin, S. D., Rothlein, R., Toman, C., and Anderson, D. C. (1989). Cooperative interactions of LFA-1 and Mac-1 with intercellular adhesion molecule-1 in facilitating adherence and transendothelial migration of human neutrophils in vitro. J. Clin. Invest. 83, 2008-2017. doi: 10.1172/JCI114111

Soares, R. P., Xander, P., Costa, A. O., Marcilla, A., Menezes-Neto, A., Del Portillo, H., et al. (2017). Highlights of the São Paulo ISEV workshop on extracellular vesicles in cross-kingdom communication. J. Extracell Vesicles 6:1407213. doi: 10.1080/20013078.2017.1407213

Theelen, B., Cafarchia, C., Gaitanis, G., Bassukas, I. D., Boekhout, T., Dawson, T. L., et al. (2018). Malassezia ecology, pathophysiology, and treatment. Med. Mycol. 56 (Suppl_1):S10-S25. doi: 10.1093/mmy/myx134

Thery, C., Witwer, K. W., Aikawa, E., Alcaraz, M. J., Anderson, J. D., Andriantsitohaina, R., et al. (2018). Minimal information for studies of extracellular vesicles 2018 (MISEV2018): a position statement of the International Society for Extracellular Vesicles and update of the MISEV2014 guidelines. J. Extracell Vesicles 7:1535750. doi: 10.1080/20013078.2018.1535750

Vallhov, H., Gutzeit, C., Hultenby, K., Valenta, R., Gronlund, H., and Scheynius, A. (2015). Dendritic cell-derived exosomes carry the major cat allergen Fel $\mathrm{d} 1$ and induce an allergic immune response. Allergy 70, 1651-1655. doi: $10.1111 /$ all.12701

Watanabe, S., Kano, R., Sato, H., Nakamura, Y., and Hasegawa, A. (2001). The effects of Malassezia yeasts on cytokine production by human keratinocytes. J. Invest. Dermatol. 116, 769-773. doi: 10.1046/j.1523-1747.2001.01321.x

Woith, E., Fuhrmann, G., and Melzig, M. F. (2019). Extracellular vesiclesconnecting kingdoms. Int. J. Mol. Sci. 20:5695. doi: 10.3390/ijms20225695

Zhang, Y.-J., Han, Y., Sun, Y.-Z., Jiang, H.-H., Liu, M., Qi, R.-Q., et al. (2019). Extracellular vesicles derived from Malassezia furfur stimulate IL-6 production in keratinocytes as demonstrated in in vitro and in vivo models. J. Dermatol. Sci. 93, 168-175. doi: 10.1016/j.jdermsci.2019.03.001

Conflict of Interest: AS is a member in the Joint Steering Committee for the Human Translational Microbiome Program at SciLifeLab/Karolinska Institutet together with Ferring Pharmaceuticals, Switzerland.

The remaining authors declare that the research was conducted in the absence of any commercial or financial relationships that could be construed as a potential conflict of interest.

Copyright $\odot 2020$ Vallhov, Johansson, Veerman and Scheynius. This is an openaccess article distributed under the terms of the Creative Commons Attribution License (CC BY). The use, distribution or reproduction in other forums is permitted, provided the original author(s) and the copyright owner(s) are credited and that the original publication in this journal is cited, in accordance with accepted academic practice. No use, distribution or reproduction is permitted which does not comply with these terms. 